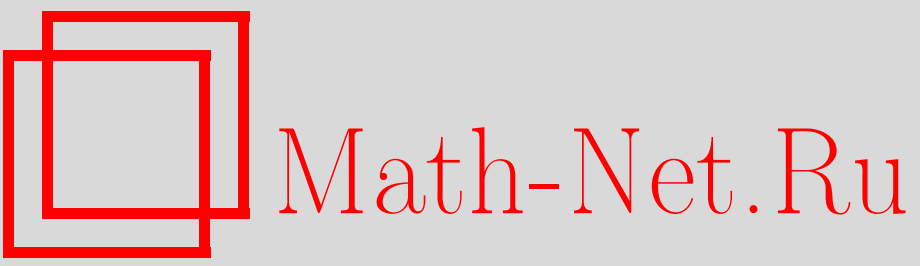

Г. Каниадакис, П. Кварати, А. М. Скарфоне, Статические вихревые решения для расположенной на плоскости системы частиц, подчиняющейся обобщенному принципу Паули, ТМФ, 2001, том 127, номер 3, 411-417

DOI: https://doi.org/10.4213/tmf469

Использование Общероссийского математического портала Math-Net.Ru подразумевает, что вы прочитали и согласны с пользовательским соглашением

http://www.mathnet.ru/rus/agreement

Параметры загрузки:

IP: 54.209 .52 .79

26 апреля 2023 г., 15:40:52 
ТЕОРЕТИЧЕСКАЯ

И МАТЕМАТИЧЕСКАЯ

ФИЗИКА

Том 127, № 3

июнь, 2001

\section{(c) 2001 г. $\quad$ Г. Каниадакис* СТАТИЧЕСКИЕ ВИХРЕВЫЕ РЕШЕНИЯ ДЛЯ РАСПОЛОЖЕННОЙ НА ПЛОСКОСТИ СИСТЕМЫ ЧАСТИЦ, ПОДЧИНЯЮЩЕЙСЯ ОБОБЩЕННОМУ ПРИНЦИПУ ПАУЛИ}

Рассматривается система частиц на плоскости, подчиняющаяся обобщенному принципу Паули. В приближении среднего поля система описывается недавно предложенным нами уравнением Шредингера с комплексной нелинейностью. В такой системе сохраняется число частиц, полная энергия и угловой момент. Рассматриваются вихреподобные стационарные конфигурации вида $\psi(\mathbf{r})=\rho(r)^{1 / 2} e^{i n \theta}$ и записывается дифференциальное уравнение, определяющее форму вихря. Найдено аналитическое решение этого уравнения и получено замкнутое выражение для профиля вихря. Исследуются некоторые средние характеристики системы, в частности, вычисляются энергетический спектр и угловой момент вихря.

С первых лет развития квантовой механики, конкретно с 1932 г. [1], стало ясно, что эффекты статистики, обусловленные принципом Паули для системы свободных фермионов, можно воспроизвести, вводя межчастичный отталкивательный потенциал взаимодействия в координатном пространстве. В начале 50-х годов Грин показал [2], что принципы квантовой механики допускают два вида статистик, являющихся обобшениями известных бозе- и ферми-статистик и названных соответственно пара-бозе- и пара$\oint е р$ ми-статистиками. С тех пор неоднократно предлагались другие обобшенные статистики [3]-[5] для многочастичных квантовых систем с целью объяснения некоторых эффектов, наблюдавшихся в физике конденсированных сред [6]-[8].

В работе [9] нами был проквантован марковский процесс, подчиняюшийся обобшенному принципу Паули ("exclusion-inclusion principle") в координатном пространстве. Динамика канонической квантовой многочастичной системы, подчиняюшейся такому принципу, описывается нелинейным уравнением Шредингера в $(D+1)$-мерном координатном пространстве:

$$
i \hbar \frac{\partial \psi}{\partial t}=-\frac{\hbar^{2}}{2 m} \Delta \psi+W(\rho, \mathbf{j}) \psi+i \mathcal{W}(\rho, \mathbf{j}) \psi+V \psi,
$$

*Dipartimento di Fisica, Politecnico di Torino, Corso Duca degli Abruzzi 24, I-10129 Torino, Italy and Istituto Nazionale di Fisica della Materia, Unitá del Politecnico di Torino, Corso Duca degli Abruzzi 24, I-10129 Torino, Italy. E-mail: kaniadakis@polito.it; quarati@polito.it; scarfone@polito.it 
где действительная и мнимая части нелинейности соответственно имеют вид

$$
\begin{aligned}
& W(\rho, \mathbf{j})=\kappa \frac{m}{\rho}\left(\frac{\mathbf{j}}{1+\kappa \rho}\right)^{2}, \\
& \mathcal{W}(\rho, \mathbf{j})=-\kappa \frac{\hbar}{2 \rho} \nabla \cdot\left(\frac{\mathbf{j} \rho}{1+\kappa \rho}\right) .
\end{aligned}
$$

Константа $\kappa$ - свободный параметр, определяюший силу эффектов запрета, a $\nabla \equiv$ $\left(\partial / \partial x^{1}, \ldots, \partial / \partial x^{D}\right)$ и $\Delta=\boldsymbol{\nabla} \cdot \boldsymbol{\nabla}-D$-мерные оператор градиента и оператор Лапласа, соответственно. Уравнение (1) может быть получено из вариационного принципа $\delta \mathcal{A} / \delta \psi^{*}=0$ с действием $\mathcal{A}=\int \mathcal{L} d^{D} x d t$, где плотность лагранжиана имеет вид

$$
\mathcal{L}=i \frac{\hbar}{2}\left(\psi^{*} \frac{\partial \psi}{\partial t}-\psi \frac{\partial \psi^{*}}{\partial t}\right)-\frac{\hbar^{2}}{2 m}|\nabla \psi|^{2}-U_{\mathrm{EIP}}\left(\psi^{*}, \psi\right)-V \psi^{*} \psi
$$

Здесь

$$
U_{\mathrm{EIP}}\left(\psi^{*}, \psi\right)=-\kappa \frac{\hbar^{2}}{8 m}\left(\psi^{*} \nabla \psi-\psi \nabla \psi^{*}\right)^{2}
$$

- нелинейньй потенциал, предназначенный для описания эффектов коллективного взаимодействия в $D$-мерной многочастичной системе, обусловленных обобшенным принципом Паули.

Мы рассматриваем случай $\kappa=-|\kappa|<0$, когда обобщенный принцип Паули сводится к обычному принципу запрета Паули. Легко видеть, что система, описываемая уравнением (1), удовлетворяет уравнению непрерывности

$$
\frac{\partial \rho}{\partial t}+\nabla \cdot \mathbf{j}=0
$$

где $\rho=|\psi|^{2}$, а квантовый ток $\mathbf{j}$ имеет вид

$$
\mathbf{j}=-\frac{i \hbar}{2 m}(1-|\kappa| \rho)\left(\psi \nabla \psi^{*}-\psi^{*} \nabla \psi\right) .
$$

Уравнение (2) обеспечивает сохранение числа частиц $N=\int \rho d^{D} x$ в системе.

Поясним кратко, как возникает уравнение (3) и каков его физический смысл. Рассмотрим марковский процесс, описываюший кинетику $N$ частиц на одномерной решетке. Узлы решетки занумеруем индексом $i$, а положение $i$-го узла обозначим через $x_{i}=$ $i \Delta x$, где $\Delta x$ - расстояние между соседними узлами. Обозначим через $\rho_{i}$ вероятность заполнения $i$-го узла и предположим, что возможны переходы только между ближайшими соседями. Процесс описывается мастер-уравнением Паули:

$$
\frac{d \rho_{i}(t)}{d t}=\pi_{i-1}^{+}(t)+\pi_{i+1}^{-}(t)-\pi_{i}^{+}(t)-\pi_{i}^{-}(t)
$$

Вероятности $\pi_{i}^{ \pm}(t)$ перехода с узла $i$ на узлы $i \pm 1$ определяются следуюшим образом:

$$
\pi_{i}^{ \pm}(t)=\frac{c_{i}^{ \pm}(t)}{(\Delta x)^{2}} \rho_{i}(t)\left[1-|\kappa| \rho_{i \pm 1}(t)\right]
$$

где $c_{i}^{ \pm}(t)$ - зависяшие от времени скорости переходов. Множители $1-|\kappa| \rho_{i \pm 1}$ учитывают коллективные эффекты, обусловленные принципом Паули. Действительно, если 
СТАТИЧЕСКИЕ ВИХРЕВЫЕ РЕШЕНИЯ ДЛЯ СИСТЕМЫ ЧАСТИЦ НА ПЛОСКОСТИ...

соседний узел не заселен, $\rho_{i \pm 1}(t)=0$, вероятность перехода $\pi_{i}^{ \pm}(t)$ зависит только от заселенности исходного узла. Если соседний узел заселен, $0<\rho_{i \pm 1}(t) \leqslant \rho_{\max }$, то вероятность перехода уменьшается. Область допустимых значений параметра $\kappa$ ограничена условием, что $\pi_{i}^{ \pm}$должны быть действительными и положительными, как и константы $c_{i}^{ \pm}$. В таком случае $|\kappa|$ лежит в области $0 \leqslant|\kappa| \leqslant 1 / \rho_{\max }$. Если определить ток частиц выражением

$$
j_{i}(t)=\left[\pi_{i-1}^{+}(t)-\pi_{i}^{-}(t)\right] \Delta x,
$$

то мастер-уравнение (4) принимает вид

$$
\frac{\partial \rho_{i}(t)}{\partial t}+\frac{\Delta j_{i}(t)}{\Delta x}=0
$$

где

$$
\Delta j_{i}(t)=j_{i+1}(t)-j_{i}(t) .
$$

В пределе $\Delta x \rightarrow 0$ получаем прямое уравнение Фоккера-Планка

$$
\frac{\partial}{\partial t} \rho(t, x)+\frac{\partial}{\partial x} j(t, x)=0
$$

где прямой ток $j(t, x)$ имеет вид

$$
j(t, x)=u(t, x) \rho(t, x)[1-|\kappa| \rho(t, x)]+\mathcal{D} \frac{\partial}{\partial x} \rho(t, x),
$$

$u(t, x)$ и $\mathcal{D}$ - коэффициенты дрейфа и диффузии, соответственно.

Рассматриваемый стохастический марковский процесс очевидным образом несимметричен по времени. Поэтому вместо прямого уравнения Фоккера-Планка (5) мы должны рассмотривать как прямое $(+)$, так и обратное $(-)$ уравнения ФоккераПланка [10]:

$$
\frac{\partial}{\partial t} \rho(t, x)+\frac{\partial}{\partial x} j^{ \pm}(t, x)=0,
$$

где прямой и обратный токи $j^{ \pm}(t, x)$ определяются следующим образом:

$$
j^{ \pm}(t, x)=u^{ \pm}(t, x) \rho(t, x)[1-|\kappa| \rho(t, x)] \pm \mathcal{D} \frac{\partial}{\partial x} \rho(t, x) .
$$

Если ввести средний ток $j(t, x)$,

$$
j(t, x)=\frac{1}{2}\left[j^{+}(t, x)+j^{-}(t, x)\right]
$$

и среднюю скорость $u(t, x)$,

$$
u(t, x)=\frac{1}{2}\left[u^{+}(t, x)+u^{-}(t, x)\right]
$$

то легко видеть, что сумма прямого и обратного уравнений Фоккера-Планка (5) и (6) дает уравнение непрерывности:

$$
\frac{\partial}{\partial t} \rho(t, x)+\frac{\partial}{\partial x}\{u(t, x) \rho(t, x)[1-|\kappa| \rho(t, x)]\}=0,
$$


а ток $j(t, x)$ оказывается чисто дрейфовым и принимает вид

$$
j(t, x)=u(t, x) \rho(t, x)[1-|\kappa| \rho(t, x)] .
$$

Обобшение уравнения (7) на случай $D$ измерений очевидно. Следуя стандартной процедуре линейной квантовой механики, запишем токовую скорость $u(t, x)$ в виде

$$
\mathbf{u}(t, \mathbf{x})=\frac{1}{m} \nabla S(t, \mathbf{x}),
$$

где $S(t, \mathbf{x})$ - фаза волновой функции $\psi=\rho^{1 / 2} e^{i S / \hbar}$. Отсюда немедленно получаются уравнения (2) и (3), причем квантовая скорость определяется соотношением

$$
\mathbf{v}(t, \mathbf{x})=\frac{1}{m}(1-|\kappa| \rho) \nabla S,
$$

а квантовый ток принимает вид

$$
\mathbf{j}(t, \mathbf{x})=\mathbf{v}(t, \mathbf{x}) \rho(t, \mathbf{x}) .
$$

Система, описываемая уравнением (1), рассматривалась в работе [9]. В частности, в случае $D=1$ были проанализированы форма и главные свойства солитонных решений уравнения (1). В данной работе рассматривается другое важное свойство этой системы, характерное для случая $D=2$. Именно, рассматривается статическое вихревое решение уравнения (1) на плоскости в свободном случае, когда потенциал $V=0$. Будем искать решение $\psi$ в виде

$$
\psi(r, \theta)=\rho(r)^{\frac{1}{2}} e^{i \mathcal{S}(\theta)},
$$

где $r=\sqrt{x^{2}+y^{2}}$ и $\theta=\operatorname{arctg}(y / x)$ - полярные координаты на плоскости, а $\mathcal{S}=S / \hbar$. Квантовая скорость $\mathbf{v}$ принимает вид

$$
\mathbf{v}(t, \mathbf{x})=\frac{\hbar}{m r} \frac{\partial \mathcal{S}}{\partial \theta}(1-|\kappa| \rho) \hat{\mathbf{e}}_{\theta}
$$

где $\hat{\mathbf{e}}_{\theta}$ - единичный вектор, ортогональньй вектору $\mathbf{x}=(x, y)$. При таком виде тока уравнение непрерывности (2) в стационарном случае удовлетворяется, только если фаза $S$ линейна по $\theta$, т.е.

$$
\mathcal{S}(\theta)=n \theta,
$$

где индекс $n \in \mathbb{N}$, чтобы поле $\psi$, определяемое формулой (8), было однозначной функцией. Квантовая скорость принимает вид

$$
\mathbf{v}(t, \mathbf{x})=\frac{\hbar}{m} \frac{n}{r}(1-|\kappa| \rho) \hat{\mathbf{e}}_{\theta} .
$$

Подставляя (8) в уравнение (1) и используя (9), получаем уравнение

$$
2 \frac{n^{2}}{r^{2}}(1-2|\kappa| \rho)=\left[\frac{1}{r \rho} \frac{d}{d r}\left(r \frac{d \rho}{d r}\right)-\frac{1}{2}\left(\frac{1}{\rho} \frac{d \rho}{d r}\right)^{2}\right] .
$$

В терминах безразмерной переменной

$$
z=\ln \frac{r}{r_{n}}
$$


СТАТИЧЕСКИЕ ВИХРЕВЫЕ РЕШЕНИЯ ДЛЯ СИСТЕМЫ ЧАСТИЦ НА ПЛОСКОСТИ...

оно принимает вид

$$
2 n^{2}(1-2|\kappa| \rho)-\frac{1}{\rho} \frac{d^{2} \rho}{d z^{2}}+\frac{1}{2}\left(\frac{1}{\rho} \frac{d \rho}{d z}\right)^{2}=0
$$

Подстановкой

$$
y(\rho)=\left(\frac{1}{\rho} \frac{d \rho}{d z}\right)^{2}
$$

последнее уравнение сводится к линейному дифференциальному уравнению первого порядка

$$
\frac{d y}{d \rho}+\frac{y}{\rho}-\frac{4 n^{2}}{\rho}(1-2|\kappa| \rho)=0,
$$

которое легко интегрируется:

$$
y(\rho)=\frac{A}{\rho}+4 n^{2}(1-|\kappa| \rho)
$$

$A$ - постоянная интегрирования. Сравнивая (11) и (12), получаем обыкновенное дифференциальное уравнение первого порядка

$$
\left(\frac{d \rho}{d z}\right)^{2}=A \rho+4 n^{2} \rho^{2}(1-|\kappa| \rho),
$$

которое можно проинтегрировать и тем самым получить форму вихря. Обозначая $f=$ $|\kappa| \rho$, мы можем представить решение уравнения (13) в неявной форме:

$$
r=r_{n} \exp \left\{ \pm \frac{1}{2 n} \int_{|\kappa| \rho_{0}}^{|\kappa| \rho} \frac{d f}{\sqrt{\alpha f+f^{2}(1-f)}}\right\}
$$

где $\alpha=A /(2 n|\kappa|)^{2}$ - произвольная постоянная. Физические вихревые решения должны быть несингулярными, неотрицательными и нормируемыми; они получаются из уравнения (14) при подходящем выборе параметров $n \in \mathbb{N}$ и $\alpha \in \mathbb{R}$.

Рассмотрим решение уравнения (14), отвечающее случаю $\alpha=0$. Легко получить явное выражение для формы вихря:

$$
\rho=\frac{4}{|\kappa|}\left[\left(\frac{r}{r_{n}}\right)^{n}+\left(\frac{r_{n}}{r}\right)^{n}\right]^{-2} .
$$

Свободный параметр $r_{n}$ связан с константой нормировки

$$
N=2 \pi \int_{0}^{\infty} \rho(r) r d r
$$

и дается формулой

$$
r_{n}=\frac{n}{2 \pi} \sqrt{|\kappa| N \sin \frac{\pi}{n}}, \quad \forall n>1 .
$$

Параметр $r_{n}$ представляет собой расстояние от центра вихря, на котором $\rho$ достигает максимума, $\rho=\rho_{\max }$. В согласии с принципом Паули имеем

$$
0 \leqslant \rho \leqslant \frac{1}{|\kappa|}, \quad \forall r \in \mathbb{R}_{0}^{+} .
$$


Более того, из формулы (15) следует, что одинарный вихрь $(n=1)$ ненормируем, поэтому одинарный вихрь является недопустимым.

Определим завихренность вихря формулой

$$
\mathcal{V}=\nabla \times \mathbf{v}
$$

где $\mathbf{v}$ дается формулой (10). С учетом выражения для профиля $\rho(r)$ вихря завихренность имеет вид

$$
\mathcal{V}=\frac{2 \pi \hbar n}{m} \delta^{(2)}(\mathbf{x})-\frac{8 \pi \hbar n^{2}}{m r_{n}^{2}}\left(\frac{r}{r_{n}}\right)^{2(n-1)}\left[1-\left(\frac{r}{r_{n}}\right)^{2 n}\right]\left[1+\left(\frac{r}{r_{n}}\right)^{2 n}\right]^{-3}
$$

и обращается в нуль на окружности $r=r_{n}$. Легко проверить, что

$$
\int_{\mathcal{R}_{2}} \mathcal{V} d^{2} x=\frac{2 \pi \hbar n}{m}
$$

тогда как интегрирование (10) по бесконечно удаленной окружности $\gamma_{\infty}$ с учетом того, что $\rho(\infty)=0$, дает

$$
\oint_{\gamma_{\infty}} \mathbf{v} \cdot d \boldsymbol{\ell}=\frac{2 \pi \hbar n}{m}
$$

Заметим, что

$$
\oint_{\gamma_{\infty}} \mathbf{v} \cdot d \boldsymbol{\ell}=\int_{\mathcal{R}_{2}} \mathcal{V} d^{2} x
$$

как и должно быть согласно определению (16). Выражение (18) оправдывает название $n$ как индекса завихренности.

Обсудим теперь средние физические характеристики вихря. Эти величины получаются исходя из общего выражения для тензора энергии-импульса системы (1), приведенного в работе [11]. Разумеется, поскольку вихри являются статическими решениями, их импульс равен нулю,

$$
\mathbf{P}=-i \frac{\hbar}{2} \int\left(\psi^{*} \boldsymbol{\nabla} \psi-\psi \boldsymbol{\nabla} \psi^{*}\right) d^{2} x=0
$$

Заметим, что уравнение (1) не является галилеево-инвариантным [11], поэтому движущиеся решения нельзя получить с помощью буста статических решений.

Угловой момент вихря, который в двумерном случае является скаляром и которому отвечает оператор $\hat{L}=-i \hbar \partial / \partial \theta$, принимает квантованные значения, пропорциональные индексу завихренности:

$$
L=n L_{0}, \quad n \geqslant 2,
$$

где

$$
L_{0}=\hbar N
$$

Заметим, что угловой момент не зависит от параметра $\kappa$.

Энергия системы $E=H$ вычисляется по гамильтониану, приведенному в работе [9]:

$$
H=\int\left[\frac{\hbar^{2}}{2 m}|\nabla \psi|^{2}+U_{\operatorname{EIP}}\left(\psi^{*}, \psi\right)\right] d^{2} x
$$


Подставляя выражение для поля $\psi$ вихря,

$$
\psi(r, \theta)=\frac{2}{\sqrt{|\kappa|}}\left[\left(\frac{r}{r_{n}}\right)^{n}+\left(\frac{r_{n}}{r}\right)^{n}\right]^{-1} e^{i n \theta},
$$

в (19), находим, что энергия вихря пропорциональна индексу завихренности:

$$
E=n E_{0}, \quad n \geqslant 2 \text {, }
$$

где

$$
E_{0}=\frac{4 \pi \hbar^{2}}{3 m|\kappa|}
$$

Нижняя гранища энергии равна $2 E_{0}$ и зависит от параметра $|\kappa|$. После несложных вычислений для дисперсии радиальной компоненты импульса получаем

$$
\left(\Delta P_{r}\right)^{2}=\frac{8 \pi \hbar^{2} n}{3|\kappa| N}
$$

а для дисперсии локализации по радиусу получаем

$$
(\Delta r)^{2}=|\kappa| N\left(\frac{n}{2 \pi}\right)^{2}\left[\frac{\sin (\pi / n)}{\cos (\pi / n)}-\frac{9}{4} \frac{\sin ^{3}(\pi / n)}{\sin ^{2}(3 \pi /(2 n))}\right] .
$$

Заметим, что для низшего $(n=2)$ вихря $\Delta r=\infty$. При $n \rightarrow \infty$ получаем $\Delta P_{r}=\infty$ и $\Delta r=0$. Наконец, легко проверить, комбинируя (21) и (22), что выполняется соотношение неопределенности

$$
\Delta P_{r} \Delta r \geqslant \frac{\hbar \pi}{3 \sqrt{2}}, \quad \forall n \geqslant 2 .
$$

\section{Список литературы}

[1] G. E. Uhlenbeck, L. Gropper. Phys. Rev. 1932. V. 41. P. 79.

[2] H. S. Green. Phys. Rev. 1953. V. 90. P. 270.

[3] F. Wilczek. Phys. Rev. Lett. 1982. V. 48. P. 114; 1982. V. 49. P. 957.

[4] O. W. Greenberg. Phys. Rev. Lett. 1990. V. 64. P. 705; Phys. Rev. D. 1991. V. 43. P. 4111.

[5] F. D. M. Haldane. Phys. Rev. Lett. 1991. V. 67. P. 937.

[6] R. B. Langhulin. Phys. Rev. B. 1983. V. 32. P. 3383.

[7] Y. H. Chen, F. Wilczek, E. Witten, B. I. Halperin. Int. J. Mod. Phys. B. 1989. V. 3. P. 1001

[8] F. Wilczek. Fractional Statistics and Anyon Superconductivity. Singapore: World Scientific, 1990.

[9] G. Kaniadakis, P. Quarati, A. M. Scarfone. Phys. Rev. E. 1998. V. 58. P. 5574.

[10] G. Kaniadakis. Phys. Rev. A. 1997. V. 55. P. 941.

[11] G. Kaniadakis, P. Quarati, A. M. Scarfone. Rep. Math. Phys. 1999. V. 44. P. 127. 